\title{
A strong root for stem cells
}

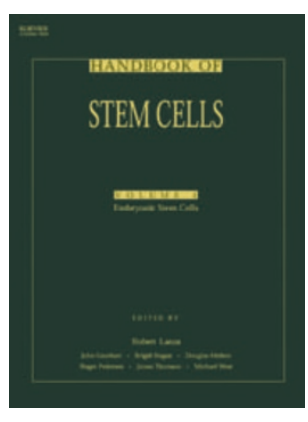

\section{Handbook on Stem Cells, Volumes 1 and 2}

Edited by Robert Lanza

Elsevier Inc. • 2004

$\$ 449.95 / £ 299.95$

\section{Steve Goldman}

The definition of a stem cell - like that of beauty — lies in the eyes of the beholder. Do stem cells strictly refer to embryonic stem cells and primordial germ cells - the pluripotent and self-renewing derivatives of blastocysts and embryonic gonads? Or should we include the multipotent but tissue-specified precursors of fetal organogenesis? Certainly the rare pluripotent cells of adult organs should be included, if they exist. But what about the organ-delimited multipotent progenitors of adult tissues? Are they too stem cells? What criteria do we use to make these claims, and what is the biological significance of the distinctions between these phenotypes?

The Handbook of Stem Cells, edited by Robert Lanza and colleagues, is an ambitious new text that achieves extraordinary completeness and inclusiveness among these different claimants to the stem cell mantle. The two-volume handbook takes an expansive approach to organizing contemporary knowledge in stem cell biology. That it generally does so effectively and fluidly is a testament to its exceedingly strong roster of editors. Because it has been compiled by many authors, the handbook does occasionally wander in its focus; but with 13 listed editors, an editorial board of 29, and a cast of 330 authors, its organizational challenges were no doubt formidable. Nonetheless, the editors have succeeded in putting together a reference that is broad enough in scope, but sufficiently detailed and rigorous, to be of real interest to both new and seasoned investigators in the field.

Both volumes are organized as distinct compendiums, with each structured to mirror the categories covered by the other. Each volume is thus ordered into sections comprising basic stem cell biology, early development, the specific derivatives of the three germinal layers, methods, applications and regulations. This parallel structure allows each volume to stand alone, although at the expense of some redundancy between them. This is especially apparent in the sections on basic biological mechanisms, which suffer frequent inter-volume overlap. However, these are also among the most well written and exciting chapters in both volumes. Indeed, had these chapters been combined and integrated, they would alone have presented a formidable handbook on stem cell biology.

Steve Goldman is in the Department of Neurology, Division of Cell and

Gene Therapy, University of Rochester Medical Center, 601 Elmwood

Avenue/MRBX, Box 645, Rochester, NY 14642, USA.

e-mail:Steven_Goldman@urmc.rochester.edu
The first volume covers embryonic stem cell biology, and is the more uniform of the two. It comprises a consistent and extraordinarily strong group of authors, a well organized framework and often superb expository writing. Many fundamentally difficult issues in basic stem cell biology - such as the basis for pluripotency, chromatin modification, developmental transcriptional regulation and epigenetic reprogramming - are presented with both clarity and insight. Subsequent chapters on ectodermal, mesodermal and endodermal embryonic stem cell derivates are presented in a logical sequence such that observations in one system inform those made in others. The methods section, although less thematically organized than other sections, is full of broad insight into what is possible; these chapters will prove valuable not only to new stem cell biologists, but also to experienced investigators who wish to learn about the methodologies in related fields.

\section{...this two-volume set will serve as}

\section{a benchmark reference in stem cell biology for years to come.}

The second volume covers both adult and fetal tissue-derived stem and progenitor cell biology. As the more topical of the two volumes, it is often the less cohesive. For instance, the otherwise interesting chapter on stem cell maintenance in plants appears in the methods section of volume two, when it might have been better integrated into the basic biology section; as the sole chapter on plants, it seems quite lonely where it appears. Volume two is also less consistent in quality then volume one. Its choice of authors is often curious, and some of the phenotype-specific discussions in this volume are too narrow to do justice to their fields. Nonetheless, most of the authors are superbly qualified and present broadly considered and compelling discussions of their topics.

Aside from its substantive discussion of the biology of stem cells and their derivatives, each volume covers the ethical implications of stem cell generation and use, and the regulatory environment and governmental policies thereof. Volume one even adds two chapters on patient perspectives, written by two hardly typical patients: Mary Tyler Moore and Christopher Reeve. Although the specific ethical, religious and patient perspectives discussed may be somewhat limited, the editor's decision to include these chapters is indeed wise; these days, public policy needs to be part of the education of any stem cell scientist. Stem cell scientists who ignore the ethical considerations and political ramifications of their work do so not only at their own peril, but to our common detriment. In providing this treatise, which covers the history, biology, methods and applications of stem cells, the editors and authors have succeeded in establishing a conceptual framework and a common language for the field. In so doing, they have ensured that this two-volume set will serve as a benchmark reference in stem cell biology for years to come. 